This item was submitted to Loughborough's Research Repository by the author.

Items in Figshare are protected by copyright, with all rights reserved, unless otherwise indicated.

\title{
Storage space allocation problem at inland bulk material stockyard
}

PLEASE CITE THE PUBLISHED VERSION

https://doi.org/10.1016/j.tre.2020.101856

PUBLISHER

Elsevier

VERSION

AM (Accepted Manuscript)

PUBLISHER STATEMENT

This paper was accepted for publication in the journal Transportation Research Part E: Logistics and Transportation Review and the definitive published version is available at https://doi.org/10.1016/j.tre.2020.101856

\section{LICENCE}

CC BY-NC-ND 4.0

\section{REPOSITORY RECORD}

Sun, Defeng, Ying Meng, Lixin Tang, Jiyin Liu, Baobin Huang, and Jiefu Yang. 2020. "Storage Space Allocation Problem at Inland Bulk Material Stockyard". Loughborough University.

https://hdl.handle.net/2134/14248400.v1. 


\title{
Storage space allocation problem at inland bulk material stockyard
}

\author{
Defeng Sun \\ Key Laboratory of Data Analytics and Optimization for Smart Industry (Northeastern \\ University), Ministry of China, P. R. China. Email: sundefeng@ise.neu.edu.cn. \\ Ying Meng* \\ Liaoning Engineering Laboratory of Operations Analytics and Optimization for Smart \\ Industry, Shenyang, 110819, P. R. China. Corresponding author, Email: \\ mengying@ise.neu.edu.cn \\ Lixin Tang \\ Institute of Industrial 83 Systems Engineering, Northeastern University, Shenyang, \\ 110819, P. R.China.Email: lixintang@ise.neu.edu.cn \\ Jiyin Liu \\ School of Business and Economics, Loughborough University, Leicestershire, LE113TU, \\ UK.Email: J.Y.Liu@lboro.ac.uk \\ Baobin Huang, Jiefu Yang \\ Liaoning Key Laboratory of Manufacturing system and Logistics, Shenyang, 110819, P. \\ R. China
}

\begin{abstract}
We consider the storage space allocation problem at an inland bulk stockyard, which aims to find an effective way to store the shapeless materials. Due to the specific storage and handling means in the stockyard, unloading, stacking and reclaiming operations need to be scheduled in an integrated manner. Viewing the space of each stock pad in the yard as a series of unit slots, we first develop a novel MIP formulation which can avoid generating scattered small fields. We then decompose the model by exploiting the relationships among the above operations, and develop a logic-based Benders approach to solve it optimally.
\end{abstract}

Keywords: storage space allocation, bulk material, Benders decomposition. 


\section{Introduction}

Bulk material stockyard is a centralized storage site for bulk materials such as iron ore and coal. It acts as an essential buffer which could absorb unavoidable differences between incoming and outgoing streams of bulk materials(Lodewijks et al. (2007)). Incoming materials are unloaded from transportation vehicles, stored in the stockyard, and reclaimed later when needed for use. Due to the increasing demands of bulk materials, the storage space in the bulk stockyard becomes a scarce resource in the daily operation management. On the other hand, the expansion of the stockyard requires huge investment (Van Vianen et al. (2014)). Therefore, it is essential to improve the performance of storage space allocation in the bulk material yard.

The goal of the storage space allocation for bulk materials is to find an effective way to store the shapeless materials. In contrast to the space allocation problem in container yard where we can put a standard-sized container anywhere if there is a space, in bulk material yard, we have to find a location that has enough capacity to store a certain volume of one material.

As shown in Figure 1, the bulk materials are usually stored in the form of stockpiles. The stockyard consists of several stock pads, each of which is a long strip area. At any time point, some parts of the stockyard may be occupied by existing stockpiles, leaving the other parts (called empty fields) available to stack new incoming materials. Due to the fact that these fields have different capacities, and that the materials have different volumes, it is hard to find the fields perfectly matching the materials. Space allocation decisions with poor matching will increase the risk of causing scattered small fields, which are small in capacity and are hard to be utilized in future. Considering the limited storage space in the stockyard, this could significantly reduce the storage and operation efficiency.

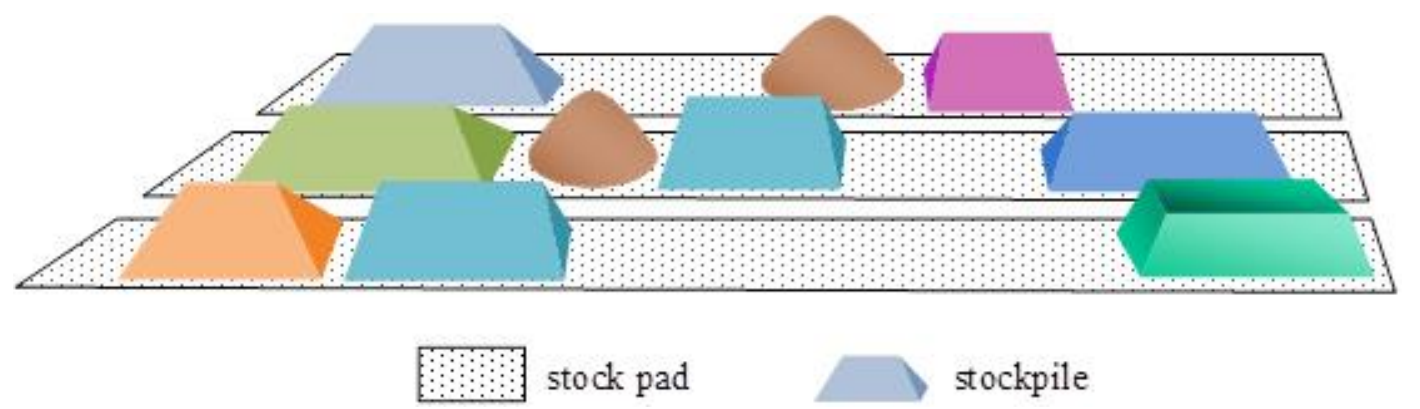

Figure 1: The view of a bulk material stockyard

The major decisions to be made in the studied storage space allocation problem is to determine the specific stacking location in the stock pads for each incoming material. However, the storage space allocation decisions cannot be made independently. The operations 
in bulk material yard are highly interdependent (see Xin et al. (2018), Unsal and Oguz (2019)). When being unloaded in an unloading station, a certain material is simultaneously transported by the belt conveyors to a stock pad and then stacked. Therefore, space allocation decisions should be made together with the scheduling decisions of unloading operations at each unloading station and the stacking operations or even the reclaiming operations in each pad, to guarantee the corresponding time window requirements of these operation tasks.

The major contributions in this paper can be stated as follows: 1) we develop a novel MIP formulation to solve the storage space allocation problem in bulk material yards, in which we tackle the practical challenge of avoiding scattered small fields for the first time. 2) we exploit the relationships among the operations to decompose the problem, and furthermore develop an efficient Benders Decomposition based approach to solve the problem optimally. The approach could take the advantages of multi-cut generation and logic-based cut generation strategies.

In the rest of the paper, we first provide a literature review in Section 2. Section 3 describes the studied problem in details and provides a novel mathematical formulation. Our formulation enables us to develop an efficient Benders Decomposition approach for its solution, which is enhanced by multi-cut generation and logic-based cut generation (Section 4). Computational studies are conducted in Section 5 to evaluate the performance of the proposed Benders Decomposition based approach. Finally, we summarize our results and conclusions in Section 6.

\section{Literature review}

Bulk material yard and container yard are two typical transit-storage systems for largevolume cargoes. They both perform typical handling operations such as unloading the cargoes from ships, train or trucks, stacking the cargoes in the yard, retrieving the cargoes from the yard and transporting the cargoes between storage and berth areas or unloading stations. However, as described above, their means of storing and handling cargoes differ vastly in terms of the types of cargoes(Unsal and Oguz (2019)). The storage operation in container yard has been extensively studied. For a detailed information on the subject, we refer readers to Zhang et al. (2003), Bazzazi et al. (2009), Wan et al. (2010), Yu and Qi (2013) and Tang et al. (2015). In contrast, studies related to bulk material yard are still limited, although there has been significant growth in bulk transport over the last few decades.

Most of the previous studies simplified the consideration of storage space allocation by allocating one material to one field (or so-called location), ignoring the capacity difference among the fields. Ago et al. (2007) developed a Lagrangian decomposition approach to 
solve the storage allocation and routing problem for the stockyard in steel-making plants, aiming at allocating the materials to each stock area under the maximum capacity limit and determining the belt-conveyor transportation routes. Barros et al. (2011) considered the stock level of the bulk materials in the stockyard when making the berth allocation decisions. Boland et al. (2012) studied the planning problem at export bulk ports, which considers the locations of the stockpiles that should be reclaimed. Robenek et al. (2014) highlighted the differences in operations between bulk ports and container terminals and derived a branch-and-price algorithm to solve the integrated berth allocation and yard assignment problem. Tang et al. (2016) was the first to explicitly consider the specific locations of the bulk materials by partitioning each stock pad into a number of slots and studied the integration of ship scheduling and storage space allocation in bulk material port. Al-Hammadi and Diabat (2017) extended the work of Robenek et al. (2014) by adding storage space capacity constraints. Menezes et al. (2017) studied an integrated production planning and scheduling problem in bulk ports, and considered the stockyard allocation from the perspective of routine selections. Unsal and Oguz (2019) considered the integrated planning problem of export dry bulk terminals which consists of the operations of berth allocation, reclaimer scheduling and stockyard allocation. They assigned the stockpile to be claimed for each moored vessel so as to minimize the departure time of the vessels.

In practice, the storage space allocation of each material has a significant influence on the future layout of the stockyard. Scattered small fields are a significant consequence of poor allocation, which should be avoided. However, in the existing modeling frameworks mentioned above, storage space allocation was considered in a discrete-event manner, and therefore, varieties of the storage capacity and changes of the fields over time were not considered. Xin et al. (2018) pointed out that the storage space in the bulk stockyard may change over time, and developed a dynamical process of material storage allocation in a rolling horizon manner based on a model predictive controller. To the best of our knowledge, the practical concern of avoiding scattered small fields has not been addressed in the previous literature.

The storage space allocation problem in bulk material yard also involve the unloader scheduling (or berth allocation) and stacker/reclaimer scheduling. These scheduling problems aim at minimizing the total time or tardiness of the operations. Umang et al. (2013) considered the berth allocation problem in bulk terminals and developed both exact and heuristic approaches to solve it. They regarded the specialized equipment facilities as cargo types in their modeling so as to avoid the unmatched allocation of berth sections and facilities to vessels. Babu et al. (2015) minimized the delay of the bulk material ships by developing two heuristic-based greedy algorithms which considered the influence of stockyard planning and train scheduling. Angelelli et al. (2016) studied the problem of 
scheduling reclaimers in the stockyard of a coal export terminal and developed a constant factor approximation algorithm to solve it. Kalinowski et al. (2017) proposed a mixedinteger formulation for the reclaimer scheduling problem in the coal stockyard. They assumed that there were two reclaimers mounted on a rail track which was located inbetween two pads. These studies focused more on the scheduling of handling equipment rather than storage space allocation.

\section{Problem description and mathematical formu- lation}

\subsection{Problem description}

In this paper, we investigate the storage space allocation problem derived from inland bulk material yards in steel enterprises. Unlike the case of the bulk material yard located at a port, the bulk materials are usually transported to the inland yard in smaller lots by trains or trucks. That is, the unloading and stacking operations of incoming materials are more frequently carried out.

Figure 2 is the schematic diagram of the storage space allocation problem we studied in this paper. For each incoming material, the incoming operation is composed of three simultaneous operations, i.e. unloading operation through the unloading equipmen$\mathrm{t}$, transportation operation through the belt conveyors and stacking operation through the stackers. Hereinafter, we denote the group of the above three operations corresponding to the same incoming material as an incoming task. Once demanded, bulk materials will be collected from the stockyard through reclaimers and transport to production sites through belt conveyors. As a common case in practice, we consider that the stacking and reclaiming operations are both carried out by the same large equipment called stacker-reclaimer(Xin et al. (2018)). We denoted it as $S R$ hereinafter. For a given stock pad, there is a unique SR installed on the trail along it(Hu and Yao (2012)). Once a certain SR starts a stacking task or a reclaiming task, it cannot be interrupted until the task is completed.

As shown in Figure 2, a stockpile uses the entire width of a stock pad but only a portion of its length(Unsal and Oguz (2019)). The region between two adjacent stockpiles is called an empty field which can be used to stack new incoming materials. Each incoming material will be stacked as a continuous stockpile separated from others. Note that the maximum amount of each material that can be allocated to a unit length of storage space is a known constant due to the fixed bulk density. Therefore, the length of the corresponding storage space needs to stack each material can be regarded as a known parameter since the total amount of each incoming material is known.

There are two types of decisions that should be made in the storage space allocation 


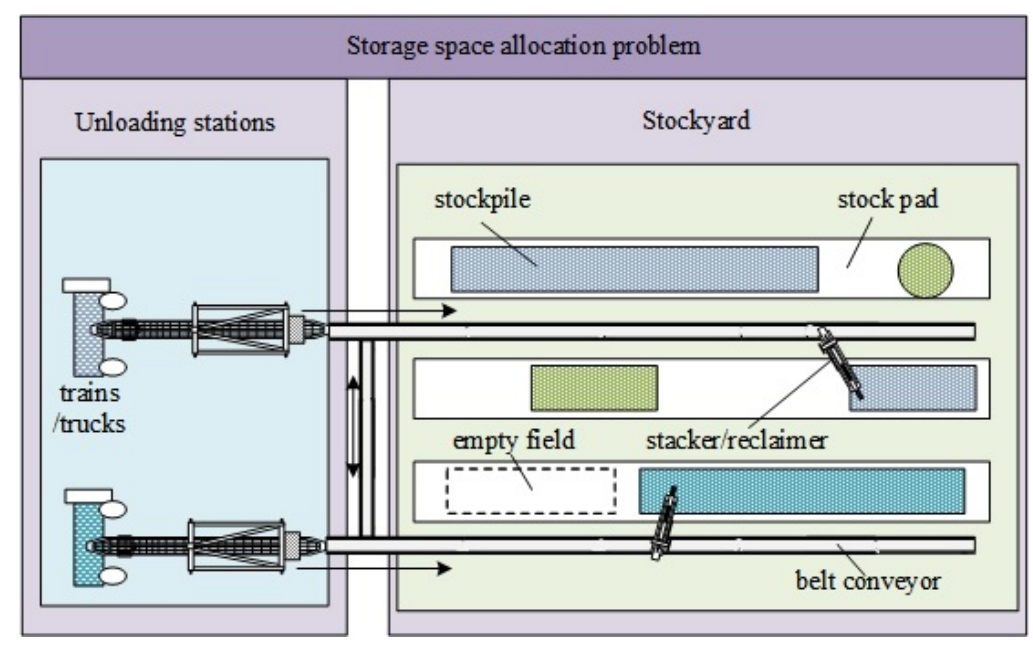

Figure 2: The storage space allocation problem at an inland bulk material stockyard

problem for bulk material stockyard. One is operation scheduling decision which determines the completion time of each incoming task, and the other is storage space allocation decision which determines the specific stacking location of each incoming material in the stock pads. These two types of decisions are coupled with each other in practice. Once a material is allocated to be stacked in a given pad, we should determine the operation sequence between the corresponding incoming task and the reclaiming tasks in the same pad since all the tasks in the pad are operated by the same SR.

As to the task scheduling decisions, the unloading precedence relationship among the incoming materials is usually pre-determined by a tradeoff strategy based on the first-infirst-out policy and the shortage-first policy. However, when the wagons get to unloading stations, the railway company will set a reasonable due leaving time for these wagons. Tardiness is allowed but a penalty will be charged by the railway company as a consequence. The operation sequence among the incoming tasks and the reclaiming tasks have a significant influence on the completion time of each incoming task, although the sequence of reclaiming tasks in a given pad is pre-determined.

To simplify the modelling of storage space allocation decisions, we introduce the concept of stock slot similar to Tang et al. (2016). We assume that one stock pad is composed of a series of unit storage spaces, called slots, each of which is 5 meters long (i.e. the length of the minimum safety distance as shown in Figure 3). For a stockpile which will not be fully reclaimed over the planning horizon (e.g. stockpiles 1 and 2 in Figure 3), the corresponding slots cannot be allocated to store incoming materials in the period and so we do not need to consider their actual size. Thus, we introduce a border slot to replace the corresponding slots, and restrict that no materials can be stacked on this border slot, like slots 4 and 7 in Figure 3(b). 
Specially, if a stockpile will be partly reclaimed from left during the planning horizon, we define that the corresponding border slot can be left-updated. For example, in Figure 3 , border slot 2 (i.e. slot 7 ) is corresponding to stockpile 2 in the original stock pad. Since stockpile 2 will be partly reclaimed from left, slot 7 is a border slot that can be left-updated. Similarly we have the border slot that can be right-updated. That is to say, we define two types of border slot, one is the general border slot like slot 4 , and the other is updatable border slot like slot 7 . The definition of updatable border slot is essential to the concern of scattered small field. For example, stockpile 2 will be reclaimed within a couple of days from the left and therefore the corresponding border slot 2 is left-updatable. In this case, we can allow the generation of scattered small field on the left side of the left-updatable border slot 2 , since a longer consecutive field will be formed and then used to accommodate a new stockpile in the near future.



(a) original stock pad

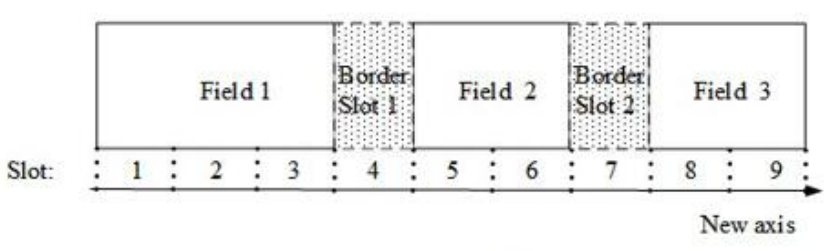

(b) new stock pad

Figure 3: The modeling of storage space in a pad

The introduction of the border slot can help reduce the problem scale and bring flexibility to the resulting formulation. For example, for the case where there are two pads being served by the same SR, we can merge the two pads into one by simply adding a special border slot between the slot groups of these two pads.

As mentioned above, we also take into account the practical consideration of scattered small fields. Let $a$ and $b$ be the lower limit and the upper limit of the length of any scattered small field. Empty field with a length smaller than $a$ can be allowed to act as the saftey distance, while empty field with a length between $a$ and $b$ inclusive is called a scattered small field which is not long enough to stack a material and will be wasted. To 
improve the space utilization of the stockyard, scattered fields should be avoided.

Figure 4 shows a simple example of the modeling structure for a scattered small field with $a=2$ and $b=5$. For a given slot $s$, we first define its forbidden regions $[s+a+1, s+b+1]$ and $[s-b-1, s-a-1]$, as well as the safety regions $[s+1, s+a]$ and $[s-a, s-1]$. Let slot 9 in Figure 4 be the given slot, there are totally two cases that we should consider the possibility of scattered small fields. The first case is that slot 9 is a border slot. Suppose that slot 9 cannot be right-updated. If a new stockpile begins from a slot with the right forbidden region of slot 9 (i.e. slots 12 to 15), a new field will be generated on the right side of slot 9 . Since the length of this new field is in the range $[a, b]$, i.e., $[2,5]$, it is a scattered small field and should be avoided. Similarly, a new stockpile should not end its stacking in a slot in the left forbidden region of slot 9 (i.e. slots 3 to 6 ) so as to avoid scatter small field on the left side when it cannot be left-updated. The second case is that slot 9 is not a border slot. In this case, we should consider the restrictions corresponding to the left (or right) forbidden region when an incoming material will start (or end) its stacking in slot 9 .

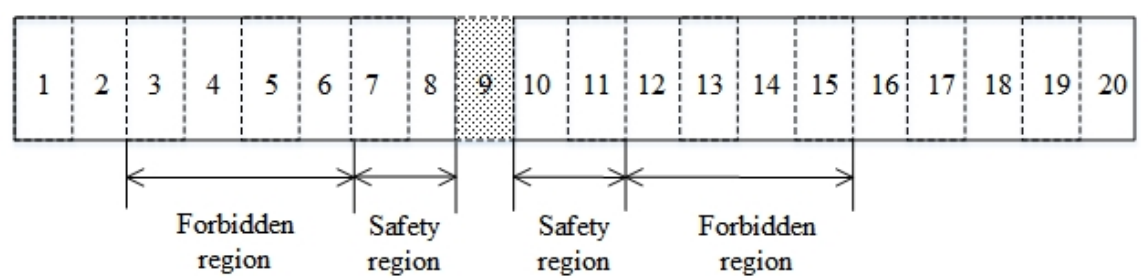

Figure 4: The modelling of scattered small field

In the given time horizon, the newly generated empty fields resulted from the reclaiming operations are not allowed to stack materials, due to the fact that the clearance operations of these new fields are time consuming, and the fact that these new fields are usually announced as available empty fields at the end of the planning horizon in practice. However, when identifying the scattered fields, these new fields are taken into consideration. That is, we can allow the generation of a scattered field which can be continuously connected with a newly generated empty field to form a longer empty field in future.

After a stockpile is reclaimed, there are still inevitable residuals left and will be mixed with the next material stacked at the same location (Tang et al. (2016)). However, in the stockyard of a steel plant, materials of different chemical components and physical particle sizes will probably be sent to different mills due to the different production process. For example, the iron ores can be roughly divided into two groups according to their particle sizes, i.e. the lump ores and the ore fines. Lump ore is sent to the blast furnace directly while the ore fine is sent to the sintering mill (after blending) or pelletizing mill first for agglomeration(Min et al. (1997)). Thus, the mixture among different ore types should be 
dealt with by extra operations such as sieving, otherwise it will be regarded as material loss. Also, it is preferred to store a type of ore in a region which was previously used to store the same type of ore for management and operation convenience. Similar concerns can also be addressed for coal, which includes coking coal, injection pulverized coal and thermal coal. Specially, in the bulk material yard we studied, the coal and ore are not allowed to be mixed, and therefore, the mixture cost between iron ore and coal is set to be extremely high.

\subsection{Model formulation}

In this section, we introduce an innovative model formulation for the bulk material storage space allocation problem, in which we take into consideration the practical requirement of avoiding scattered small fields.

Sets and parameters

$I$ : set of incoming tasks/materials;

$W$ : set of unloading stations;

$K$ : set of stock pads;

$N_{k}$ : set of the reclaiming tasks in pad $k, k \in K$; the reclaiming tasks in $N_{k}$ are numbered sequentially starting from 1 according to the required reclaiming order;

$S_{k}$ : set of the slots in pad $k, k \in K$;

$\Phi$ : set of task precedence pairs $(i, j)$ for $i \in I$ and $j \in I$;

$\alpha_{i}$ : unit penalty cost of the tardiness of incoming task $i$;

$\beta_{i k s}$ : material mixture cost when material $i$ is allocated to be stored at a location ends at slot $s$ in stock pad $k$;

$e_{i}^{1}$ : earliest start time of the incoming task $i, i \in I$;

$d_{i}^{1}$ : due completion time of the incoming task $i, i \in I$;

$e_{k n}^{2}:$ earliest start time of the reclaiming task $n$ in pad $k$;

$d_{k n}^{2}$ : due completion time of the reclaiming task $n$ in pad $k$;

$h_{k s}$ : binary parameter which indicates whether or not slot $s$ in pad $k$ is a border slot;

$g_{k s}^{L}$ : binary parameter which indicates whether or not slot $s$ in pad $k$ is a border slot that can be left-updated; note that if $g_{k s}^{L}=1$, we have $h_{k s}=1$; 
$g_{k s}^{R}$ : binary parameter which indicates whether or not slot $s$ in pad $k$ is a border slot that can be right-updated; note that if $g_{k s}^{R}=1$, we have $h_{k s}=1$;

$p_{i}^{1}$ : operation time of the incoming task $i$;

$p_{k n}^{2}$ : operation time of the reclaiming task $n$ at pad $k$;

$l_{i}$ : length of storage space needed to stack the material corresponding to incoming task $i$, including the length of an extra safety slot;

$a, b$ : the lower and upper limits, respectively, of the length of any scattered small field. By definition, a scattered small field would not be enough to store a material, so we have $b<l_{i}$ for all $i$.

\section{Decision variables}

$t_{i}$ : the tardiness time of the incoming task $i$;

$c_{i}^{1}$ : the completion time of the incoming task $i$;

$c_{k n}^{2}$ : the completion time of the reclaiming task $n$ in pad $k$;

$u_{i w}:(0-1)$ variable equal to 1 if and only if the the incoming task $i$ is unloaded in unloading station $w$;

$x_{i k n}$ : (0-1) variable equal to 1 if and only if the incoming task $i$ is operated after reclaiming task $n$ and before reclaiming task $n+1$ in stock pad $k$; Specially, $x_{i k 0}$ indicates that the incoming task $i$ is operated before all the reclaiming tasks in stock pad $k$;

$y_{i k}$ : (0-1) variable equal to 1 if and only if the incoming material $i$ is allocated to be stacked in stock pad $k$;

$z_{i k s}$ : (0-1) variable equal to 1 if and only if the stacking of incoming material $i$ ends at slot $s$ in stock pad $k$.

Observe that slot $s$ of stock pad $k$ is occupied by material $i$ if $\sum_{r=s}^{s+l_{i}-1} z_{i k r}=1$. Thus the storage space allocation problem at inland bulk material stockyard (SSAPB) can be formulated as follows:

$$
\begin{aligned}
& (S S A P B) v(S S A P B)=\min \sum_{i \in I} \alpha_{i} t_{i}+\sum_{i \in I} \sum_{k \in K} \sum_{s \in S_{k}} \beta_{i k s} z_{i k s} \\
& \text { s.t. } t_{i} \geq c_{i}^{1}-d_{i}^{1}, \quad \forall i \in I \\
& c_{i}^{1}-p_{i}^{1} \geq e_{i}^{1}, \quad \forall i \in I
\end{aligned}
$$




$$
\begin{aligned}
& e_{k n}^{2}+p_{k n}^{2} \leq c_{k n}^{2} \leq d_{k n}^{2}, \quad \forall k \in K, n \in N_{k} \\
& c_{j}^{1}-p_{j}^{1} \geq c_{i}^{1}+M \cdot\left(u_{i w}+u_{j w}-2\right), \quad \forall(i, j) \in \Phi, w \in W \\
& c_{j}^{1}-p_{j}^{1} \geq c_{i}^{1}+M \cdot\left(y_{i k}+y_{j k}-2\right), \quad \forall(i, j) \in \Phi, k \in K \\
& c_{i}^{1}-p_{i}^{1} \geq c_{k n}^{2}+M \cdot\left(x_{i k n}-1\right), \quad \forall i \in I, k \in K, n \in N_{k} \cup\{0\} \\
& c_{i}^{1} \leq c_{k, n+1}^{2}-p_{k, n+1}^{2}+M \cdot\left(1-x_{i k n}\right), \quad \forall i \in I, k \in K, 0 \leq n \leq\left|N_{k}\right|-1 \\
& c_{k, n+1}^{2} \geq c_{k n}^{2}+\sum_{i \in I} p_{i} x_{i k n}+p_{k, n+1}^{2}, \quad \forall k \in K, 0 \leq n \leq\left|N_{k}\right|-1 \\
& \sum_{w \in W} u_{i w}=1, \quad \forall i \in I \\
& \sum_{k \in K} y_{i k}=1, \quad \forall i \in I \\
& \sum_{n \in N_{k} \cup\{0\}} x_{i k n}=y_{i k}, \quad \forall i \in I, k \in K \\
& \sum_{s=l_{i}}^{\left|S_{k}\right|} z_{i k s}=y_{i k}, \quad \forall i \in I, k \in K \\
& \sum_{i \in I} \sum_{r=s}^{s+l_{i}-1} z_{i k r} \leq 1-h_{k s}, \quad \forall k \in K, s \in N_{k} \\
& \sum_{i \in I} \sum_{r=s+b+1}^{s+a+1} z_{i k, r+l_{i}-1} \leq 1-\left(\sum_{i \in I} z_{i k s}+h_{k s}-g_{k s}^{R}\right)+\sum_{r=s+1}^{s+a} \\
& h_{k r}
\end{aligned}
$$

$$
\sum_{i \in I} \sum_{r=s-b-1}^{s-a-1} z_{i k r} \leq 1-\left(\sum_{i \in I} z_{i k, s+l_{i}-1}+h_{k s}-g_{k s}^{L}\right)+\sum_{r=s-a}^{s-1} h_{k r}, \quad \forall k \in K, s \in S_{k}
$$

$x_{i k n}, y_{i k}, z_{i k s}, u_{i j} \in\{0,1\}, \quad \forall i, j \in I, k \in K, n \in N_{k}, s \in S_{k}$

$$
t_{i}, c_{i}^{1}, c_{k n}^{2} \geq 0, \quad \forall i \in I, k \in K, n \in N_{k}
$$

The objective function (1) is composed of two terms, i.e. the tardiness penalty costs, and the material mixture loss costs. Constraint (2) calculates the tardiness of the incoming operations of each material, Constraint (3) ensures each incoming task is started after its earliest available time, while constraint (4) imposes the limits of start time and completion time of each reclaiming task. Constraint (5) ensures that the completion times of the incoming tasks that unloaded at the same unloading station are in accordance with the pre-determined task precedence, while constraint (6) ensures that the incoming tasks allocated to the same pad are operated in sequence. Constraints (7), (8) and (9) calculate the completion times of the incoming tasks and the reclaiming tasks within the same stock pad. Constraint (10) ensures that each incoming task is allocated to one unloading station. 
Constraint (11) ensures that each incoming material is stacked in one stock pad. Constraint (12) states that the stacking task of material $i$ is operated between two sequential reclaiming tasks in stock pad $k$ if it is allocated to be stacked in that stock pad. Constraint (13) states that there is one and only one ending slot for material $i$ if it is allocated to be stacked in stock pad $k$. Constraint (14) ensures that at most one material can be stacked on the same slot, and that the storage space of each material cannot contain any border slot. Thus, each material is stacked in a continuous region in one stock pad, and there is no overlap between the storage location of any two materials.

Constraints (15) and (16) ensure that no scattered small field is created on the right and left sides of a material pile or a border slot, by forbidding any material to be stacked in the forbidden regions of it. Constraint (15) takes effect in the case where slot $s$ is a border slot $\left(\sum_{i \in I} z_{i k s}=1\right)$ or is the end slot of a material pile allocated $\left(h_{k s}=1\right)$ which is not to be updated $\left(g_{k s}^{R}=0\right)$, and there is not a border slot within the right-side safety region of slot $s\left(\sum_{r=s+1}^{s+a} h_{k r}=0\right)$. Under these conditions, the right hand side of the constraint will be 0 , and the constraint ensures that no material will be stacked in the right forbidden region of slot $s$. If the situation is different from the above stated, the right hand side of this constraint will be greater than 0 and the constraint will not take effect. Note that slot $s$ cannot be both a border slot and the right end of a material pile allocated, and thus the right hand side of the constraint will never be negative. Note also that if there is a border slot in the right safety region of $s$, there will not be a right forbidden region for $s$, and the right forbidden region for that border slot will be considered. Similarly, (16) ensures that no material is stacked in the forbidden region on the left side of slot $\mathrm{s}$.

\section{Benders Decomposition based approach}

Benders decomposition proposed by Benders (1962) is a partitioning procedure for solving mixed-integer programming models. It decomposes the full problem into two easier problems, i.e. an integer master problem and a linear slave problem, and then solve them in an iterative procedure. The Benders approach has been successfully applied to solve various MIP problems, such as the facility location problem (Hammad et al. (2017)), the order fulfilment problem(Li and Jia (2019)), and quay crane scheduling problem(Sun et al. (2019)).

Hooker and Ottosson (2003) extended the classical Benders approach to a logic-based form so that an arbitrary slave problem can be accommodated, such as a discrete scheduling problem. Unsal and Oguz (2019) developed a logic-based Benders approach to solve the integrated planning problem of export dry bulk terminals. The berth allocation and the yard allocation problems are separated into a master and a subproblem, respectively. The master problem is modeled with mixed-integer programming and enhanced by 
embedding relaxation of reclaimer scheduling.

As we stated before, the storage allocation problem involves two types of interrelated decisions, i.e. the operation scheduling decisions and the storage space allocation decisions. However, after exploiting the problem structure, we identify two strong relationships. The decisions of stock pad allocations (i.e. variable $y_{i k}$ ) directly affect the completion time of the incoming tasks since we have to consider operation scheduling of incoming tasks and reclaiming tasks in the same pad. Also, straightforwardly, the decisions of stock pad allocations significantly influence the storage space location of the incoming materials.

In this paper, we choose to decompose the problem into a mixed-integer scheduling master problem which determines the operation sequence and completion time of the tasks as well as the allocation of tasks to pads, and a integer allocation slave problem which is to allocate the storage space in the stock pads for the incoming materials. By doing this, we can take advantages of separable slave problem and logic-based Benders cut generation.

\subsection{Benders reformulation}

In this section, we reformulate the problem by separating the operation scheduling and the pad allocation into the master problem, and the storage space allocation into the slave problem, respectively. The variables $x_{i k n}, y_{i k}, u_{i j}, c_{i}^{1}$, and $c_{k n}^{2}$ are involved in the master problem, while the variable $z_{i k s}$ is involved in the slave problem.

Cire et al. (2016) pointed out that capturing some characteristics of the subproblem via relaxations could lead to a better performance of logic-based Benders approach. Following that, we relax the variable $z_{i k s}$ to the interval $[0,1]$ and add it into the mater problem to apply this relaxation. Denote the relaxed variable as $Z_{i k s}$. An additional variable $\pi_{k}$ is added to capture the lower-bound approximation of the material mixture cost in the objective function. Then, the benders master problem can be stated as:

$$
\begin{aligned}
(B M P) & v(B M P)=\min \quad \sum_{i \in I} \alpha_{i} t_{i}+\sum_{k \in K} \pi_{k} \\
\text { s.t. } & \text { Constraints } \quad(2)-(16) \\
& \pi_{k} \geq \sum_{i \in I} \sum_{s \in S_{k}} \beta_{i k s} Z_{i k s}, \quad \forall k \in K \\
& \sum_{s=l_{i} \mid}^{\left|S_{k}\right|} Z_{i k s}=y_{i k}, \quad \forall i \in I, k \in K \\
& \sum_{i \in I} \sum_{r=s}^{s+l_{i}-1} Z_{i k r} \leq 1-h_{k s}, \quad \forall k \in K, s \in N_{k}
\end{aligned}
$$




$$
\begin{aligned}
& \sum_{i \in I} \sum_{r=s+a+1}^{s+b+1} Z_{i k, r+l_{i}-1} \leq 1-\left(\sum_{i \in I} Z_{i k s}+h_{k s}-g_{k s}^{R}\right)+\sum_{r=s+1}^{s+a} h_{k r}, \quad \forall k \in K, s \in S_{k} \\
& \sum_{i \in I} \sum_{r=s-b-1}^{s-a-1} Z_{i k r} \leq 1-\left(\sum_{i \in I} Z_{i k, s+l_{i}-1}+h_{k s}-g_{k s}^{L}\right)+\sum_{r=s-a}^{s-1} h_{k r}, \quad \forall k \in K, s \in S_{k}
\end{aligned}
$$

$x_{i k n}, y_{i k}, u_{i j} \in\{0,1\}, 0 \leq Z_{i k s} \leq 1, c_{i}^{1}, c_{k n}^{2} \geq 0, \quad \forall i \in I, k \in K, n \in n_{k}, s \in S_{k}$

With the fixed values $\hat{y}_{i k}$ of variables $y_{i k}$, we can obtain the Benders slave problem as follows:

$$
\begin{gathered}
(S P) v(S P)=\min \quad \sum_{i \in I} \sum_{k \in K} \sum_{s \in S_{k}} \beta_{i k s} z_{i k s} \\
\text { s.t. } \sum_{s=w_{i}}^{\left|S_{k}\right|} z_{i k s}=\hat{y}_{i k}, \quad \forall i \in I, k \in K
\end{gathered}
$$

Constraints (14), (15), (16)

$$
z_{i k s} \in\{0,1\}, \quad \forall i \in I, k \in K, s \in S_{k}
$$

Let the set $I_{k}=\left\{i \in I \mid \hat{y}_{i k}=1\right\}, k \in K$. The above Benders slave problem can be separated into $|K|$ independent sub-problems which are much easier to be solved:

$$
\begin{aligned}
\left(S P_{k}\right) & v\left(S P_{k}\right)=\min \quad \sum_{i \in I} \sum_{s \in S_{k}} \beta_{i k s} z_{i k s} \\
\text { s.t. } & \sum_{s=w_{i}}^{\left|S_{k}\right|} z_{i k s}=1, \quad \forall i \in I_{k} \\
& \sum_{i \in I_{k}} \sum_{r=s}^{s+l_{i}-1} z_{i k r} \leq 1-h_{k s}, \quad \forall s \in N_{k} \\
& \sum_{i \in I_{k}} \sum_{r=s+a+1}^{s+b+1} z_{i k, r+l_{i}-1} \leq 1-\left(\sum_{i \in I_{k}} z_{i k s}+h_{k s}-g_{k s}^{R}\right)+\sum_{r=s+1}^{s+a} h_{k r}, \quad \forall s \in S_{k} \\
& \sum_{i \in I_{k}} \sum_{r=s-b-1}^{s-a-1} z_{i k r} \leq 1-\left(\sum_{i \in I_{k}} z_{i k, s+l_{i}-1}+h_{k s}-g_{k s}^{L}\right)+\sum_{r=s-a}^{s-1} h_{k r}, \quad \forall s \in S_{k} \\
& z_{i k s} \in\{0,1\}, \quad \forall i \in I, s \in S_{k}
\end{aligned}
$$




\subsection{Logic based cut}

Logic based cut is a "no-good" cut that can be applied to all types of problems(Hooker and Ottosson (2003)). It forces the master problem to eliminate the current solution and find a new solution.

Given an optimal solution $\hat{y}_{i k}$ of the master problem, there are two cases when solving each slave sub-problem, i.e. the sub-problem is feasible or infeasible. When the subproblem $S P_{k}$ is infeasible, we can draw the conclusion that the related fixed solution $\hat{y}_{i k}$ is infeasible for the full problem SSAPB too. In this case, at least one material in set $I_{k}$ should be eliminated or replaced to break the infeasibility of the related solution. Based on this observation, we derive the following logic-based Benders cut.

$$
\sum_{i \in I_{k}}\left(1-y_{i k}\right) \geq 1
$$

Given that sub-problem $S P_{k}$ under the set $I_{k}$ is infeasible. To prove that the resulted cut (37) is valid for formulation SSAPB, we have the following lemma.

Lemma 1 Any feasible solution $\left(\bar{t}_{i}, \bar{y}_{i k}, \bar{z}_{i k s}\right)$ of formulation SSAPB does not contain all the pad allocations in set $I_{k}$.

Proof. Suppose the feasible solution $\left(\bar{t}_{i}, \bar{y}_{i k}, \bar{z}_{i k s}\right)$ contains all the pad allocations in set $I_{k}$. Therefore, the corresponding sub-problem $S P_{k}$ with $I_{k}=\left\{i \in I \mid \hat{y}_{i k}=1\right\}, k \in K$ is feasible. This contradicts the initial statement that $S P_{k}$ is infeasible.

When the subproblem $S P_{k}$ is feasible, we can still derive the Benders cut base on the idea of adding relaxation information of material mixture cost into the master problem. By solving $S P_{k}$, we can obtain the minimum total material loss cost $v\left(S P_{k}\right)$ for pad $k$ under the current solution value $\hat{y}_{i k}$. Observe that, as long as that all the materials in set $I_{k}$ are allocated to be stacked in pad $k$, the resulting total material loss cost in pad $k$ will be no less than $v\left(S P_{k}\right)$. Thus, we can derive the following Benders cut.

$$
\pi_{k} \geq v\left(S P_{k}\right)-v\left(S P_{k}\right) \cdot \sum_{i \in I_{k}}\left(1-y_{i k}\right)
$$

Recall that $\pi_{k}$ is the lower bound approximation of the material mixture cost in pad $k$.

To prove that the resulted cut (38) is valid for formulation SSAPB, we have the following lemma.

Lemma 2 Any feasible solution $\left(\bar{t}_{i}, \bar{y}_{i k}, \bar{z}_{i k s}\right)$ of formulation SSAPB satisfies the following property: $\sum_{i \in I} \sum_{s \in S_{k}} \beta_{i k s} \bar{z}_{i k s} \geq v\left(S P_{k}\right)-v\left(S P_{k}\right) \cdot \sum_{i \in I_{k}}\left(1-\bar{y}_{i k}\right)$. 
Proof. If solution $\left(\bar{t}_{i}, \bar{y}_{i k}, \bar{z}_{i k s}\right)$ defines a pad allocation set different from $I_{k}$, then $\sum_{i \in I_{k}}\left(1-\bar{y}_{i k}\right) \geq$ 1 and $v\left(S P_{k}\right)-v\left(S P_{k}\right) \cdot \sum_{i \in I_{k}}\left(1-\bar{y}_{i k}\right) \leq 0$, the property holds. Otherwise, $\sum_{s \in S_{k}} \beta_{i k s} \bar{z}_{i k s}=$ $v\left(S P_{k}\right)$, the property still holds.

Benders cut (38) can be further enhanced based on the same idea of adding relaxation information of material mixture cost. Let $j$ denote a material which is not included in set $I_{k}$. If ignore all the other materials to be stacked in pad $k$, we can easily find the storage location for material $j$ with minimum material mixture cost. Denote this minimum cost as $\gamma_{j k}$, we have $\gamma_{j k}=\min _{s \in S_{k}^{j}} \beta_{j k s}, j \in I, k \in K$, where $S_{k}^{j}$ is the set of feasible slots for material $j$ in pad $k$ according to constraints (33), (34) and (35). Observe that, if we update the set $I_{k}$ by adding $j$ into it, the increased value of total material mixture costs in pad $k$ is no less than $\gamma_{j k}$. Thus, we can develop a tighter Benders cut when the sub-problem $S P_{k}$ is feasible:

$$
\pi_{k} \geq v\left(S P_{k}\right)-v\left(S P_{k}\right) \cdot \sum_{i \in I_{k}}\left(1-y_{i k}\right)+\sum_{i \in I-I_{k}} \gamma_{i k} \cdot y_{i k}
$$

We now outline the whole procedure of the proposed Benders-based approach as follows.

- Initialization. Set the lower bound $\mathrm{LB}=0$ and upper bound $\mathrm{UB}=+\infty$, set the iteration $\lambda=0$.

- While $\mathrm{LB}<\mathrm{UB}$, repeat the following procedure:

(i) Solve the master problem $B M P$, let $\left(\hat{t}_{i}, \hat{y}_{i k}\right)$ be the corresponding solution, and let $\mathrm{LB}=v(B M P)$, where $v(B M P)$ is the optimal objective value of $B M P$.

(ii) Solve each sub-problem $S P_{k}, \forall k \in K$. If $S P_{k}$ is feasible, add Benders cut (39) into BMP, otherwise, add Benders cut (37).

(iii) If all the sup-problems are feasible, update $\mathrm{UB}=\sum_{i \in I} \alpha_{i} \hat{t}_{i}+\sum_{k \in K} v\left(S P_{k}\right)$.

- Termination.

Theorem 1 The proposed Benders approach terminates with an optimal solution after a finite number of iterations.

Proof. Suppose first that the algorithm terminates with a finite solution UB. Obviously, UB is an upper bound on the optimal objective value of problem $P$. Due to Lemma 2, $\mathrm{LB}$ is a valid lower bound on the optimal objective value of problem $P$. Because LB $=\mathrm{UB}$ when the algorithm terminated, and the solution corresponding to UB is feasible, the solution is optimal. Secondly, since the domain of the master variables $y_{i k}$ is finite, only finite many subproblems can be defined (and corresponding Benders cuts), and the optimal value is reached after a finite number of iterations. 


\section{Computational experiments}

\subsection{A realistic instance}

To better illustrate the results of proposed formulation and approach, we first use the real world data from the stockyard of a steel plant in China. In the practical instance shown in Figure 5, the stockyard is composed of 9 stock pads, 5 SRs, and 2 unloading stations. The stock pads and the SRs are numbered in order. Stock pads from OA to OE are 650 meters long, while the stock pads from OF to OI are 600 meters long. There are totally 9 incoming materials waiting to be stored in the stockyard.
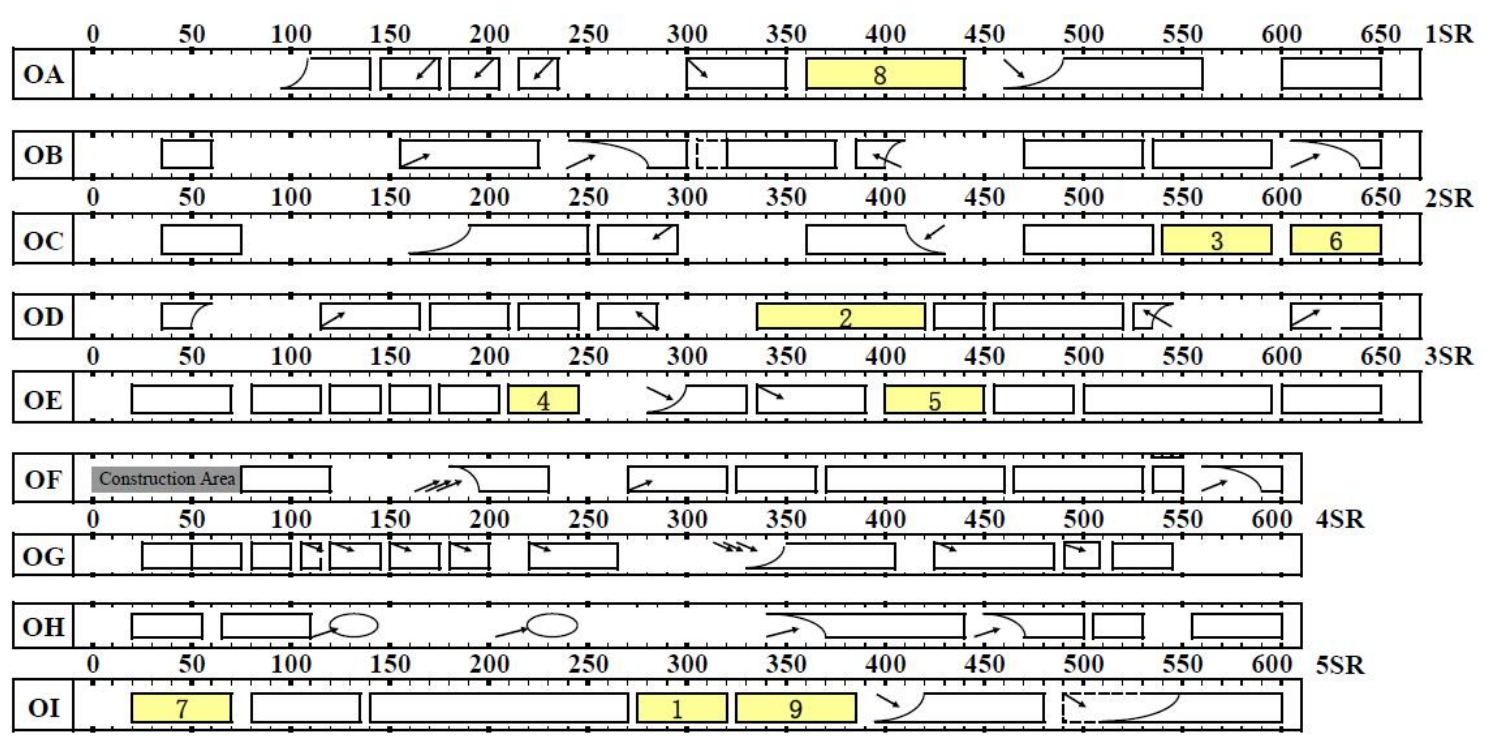

Figure 5: Storage space allocation for a stockyard that composed of 9 stock pads and 5 SRs

According to Section 3.1, we can merge two pads that served by the same SR into one new pad in our formulation. Therefore, we have totally 5 new stock pads in our formulation, i.e. OA, OB-OC, OD-OE, OF-OG and OH-OI. The stockpiles with arrows are scheduled to be fully or partly reclaimed in the planning horizon (2 days), and therefore are transformed into the updatable border slots in our formulation. The arrow indicates the planned reclaiming direction of the stockpile, and therefore tells the related border slot will be right-updated or left-updated. Other stockpiles are transformed into general border slots while empty fields are transformed into available slots, as shown in Figure 7.

When applying the Benders approach, we first solve the master problem to determine which pad should each incoming material be allocated to, as well as the operation sequence among the incoming tasks and reclaiming task in each pad. The result of the master 


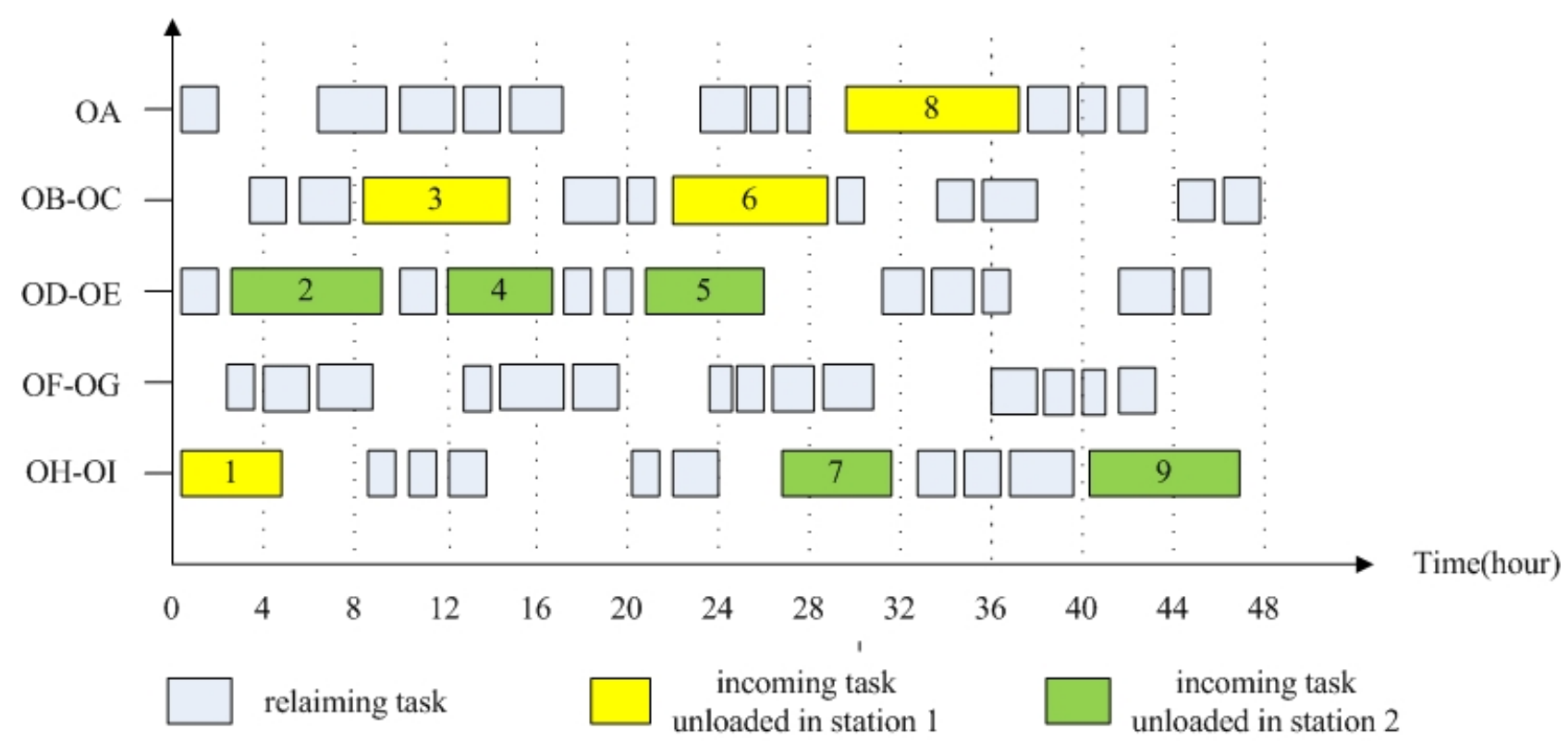

Figure 6: Result of task scheduling and pad allocation by solving master problem


a) allocation result for pad $\mathrm{OD}-\mathrm{OE}$

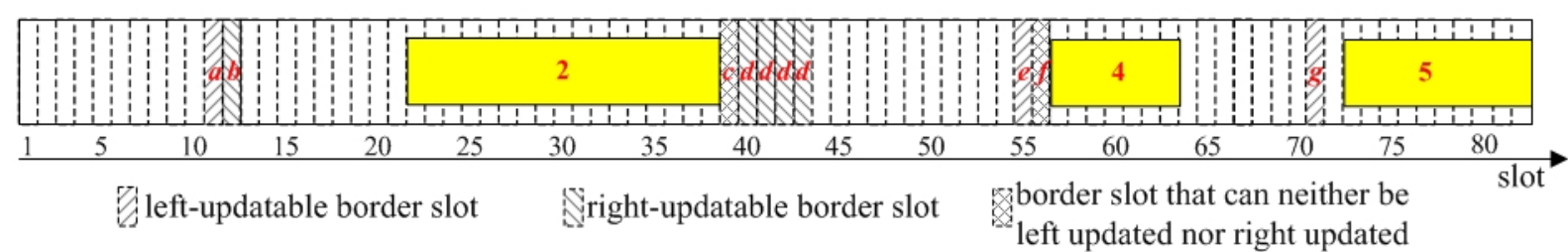

b) slot construction for sub-problem related to pad OD-OE

Figure 7: Space allocation for the sub-problem related to pad OD-OE 
problem is shown in Figure 6. Note that one stockpile may be reclaimed for multiple times in the planning horizon.

Then we can establish $|K|$ sub-problems to determine the specific locations of these materials in each pad. For example, Benders master problem has determined that incoming materials 2, 4 and 5 should be allocated to stored in the merged pad OD-OE through 3SR. A sub-problem that aims to allocate the specific location of materials 2,4 and 5 in pad OD-OE can be derived and solved. Figure 7 shows the the solution of the sub-problem related to pad OD-OE. The storage location of material 2 ends at the slot with length index 420 in pad OD, while material 4 ends at 245 and material 5 ends at 450 at pad OE.

The interval of the scattered small field we considered is set to be $[2,8]$, i.e. field length between 10 meters and 40 meters. As shown in Figure 7, no scattered small field will be generated. Specially, consider the case that material 2 is allocated to the location started from the slot with length index 550 and to the slot with length index 600 in pad OD (i.e. the field on the right of the stockpile $d$ ). Thus, a scattered small field with length of 20 meters (i.e. the length of stockpile $d$ ) will be left after stockpile $d$ is reclaimed. In this case, we consider to transform the stockpile $d$ into 4 right-updatable border slots. Thus, our formulation remains effective in preventing scattered small fields in this practical case.

\subsection{Computational results}

To verify the performance of the proposed MIP formulation and the Benders approach for the studied storage space allocation problem, computational experiments using realword data and random-generated data were conducted, respectively. To benchmark the performance, Benders master problem and slave sub-problems are all solved using the callable library of CPLEX 12.61. All the computational experiments are performed on a computer with Intel Core i7-6500U 2.50 GHz CPU and 8GB RAM.

We first conduct the experiments on 10 realistic instances given by the stockyard mentioned above. All the instances are solved to optimal by CPLEX and Benders approach, respectively. Table 1 reports the computational results of the practical instances. Column 'Deviation' reports the percent deviation between the objective function evaluation of manual scheduling and the optimal solution value of formulation SSAPB. Columns 't(CPLEX)' and 't(Benders)' are the total solution times of CPLEX and Benders approach in seconds, respectively. Column 'Dev(BMP)' reports the percent deviation between the optimal solution value of the master problem at the first iteration and that of the formulation SSAPB, to observe the performance of the master problem. Column 'iter' shows the iteration number of the Benders approach. It can be seen from Table 1 that our approach outperforms the solver CPLEX and can solve the realistic instances to optimal within a short computational time. We can observe that the proposed decomposition structure significantly reduces the number of integer variables in each formulation. The formula- 
Table 1: Performance of Benders approach based on real world data

\begin{tabular}{cccccccc}
\hline \multirow{2}{*}{ Instance } & \multicolumn{3}{c}{ CPLEX } & & \multicolumn{3}{c}{ Benders } \\
\cline { 2 - 5 } \cline { 6 - 8 } \cline { 6 - 8 } & $\mathrm{t}($ CPLEX) & Opt. & Deviation & & $\mathrm{t}$ (Benders) & Dev(BMP) & Iter \\
\hline 1 & 105.21 & 13579 & 12.72 & & 7.59 & 6.55 & 5 \\
2 & 315.80 & 9286 & 29.08 & & 2.60 & 1.03 & 3 \\
3 & 145.82 & 9911 & 16.52 & & 8.51 & 4.69 & 6 \\
4 & 362.10 & 15416 & 30.42 & & 12.33 & 5.85 & 7 \\
5 & 172.52 & 9315 & 23.30 & & 9.13 & 2.76 & 5 \\
6 & 94.19 & 3122 & 22.78 & & 7.46 & 7.32 & 4 \\
7 & 474.90 & 4320 & 18.31 & & 8.69 & 4.03 & 4 \\
8 & 163.73 & 5136 & 10.37 & & 7.05 & 5.15 & 5 \\
9 & 172.66 & 11589 & 9.42 & & 5.22 & 1.27 & 3 \\
10 & 201.58 & 8977 & 33.39 & & 6.19 & 1.08 & 5 \\
Ave. & 220.85 & & 20.63 & & 7.47 & 3.47 & 4.7 \\
\hline
\end{tabular}

tion SSAPB can promote a 20 percent cost savings on average compared with manual scheduling.

We would like to highlight the strategy of capturing some characteristics of the subproblems via relaxations in the master problem. As to our problem, the relaxation is carried out through constraints (21) to (25). Figure 8 shows that the relaxation strategy has a significant influence on the convergence rate of the Benders approach. Observe that, the solution value of the master problem (i.e. LB) at the first iteration differs significantly between the two cases. This verifies the conclusion that capturing some characteristics of the subproblem via relaxations could lead to a better performance of logic-based Benders approach.

To verify the performance of the approach under different instance scales, we carried out computational experiments on random-generated data inspired from the practice. We consider the number of materials $|I|$ in two levels 10 and 15, the number of stock pads $|K|$ in two values 5 and 7 , and the number of the slots $|S|$ in each pad in two levels 80 and 100. A triplet of $(|I|,|K|, S)$ value represents the problem size of an instances group, for which we solve 5 instances generated randomly. We consider that there are 6 border slots randomly distributed in each pad. For each pad, the number of reclaiming tasks are limited in the interval $[6,12]$. The corresponding start, operation and due times of each task are generated in a time sequence by capturing the distribution information in practice. For all the instances, the stopping criterions of a $1 \%$ optimality gap and a maximum computation time of 1800 seconds were both used.

Table 2 compares the performances of CPLEX solver and proposed Benders approach for different problem scales. The column aveT reports the average computational time of each instance group in seconds, column num reports the number of instances whose com- 


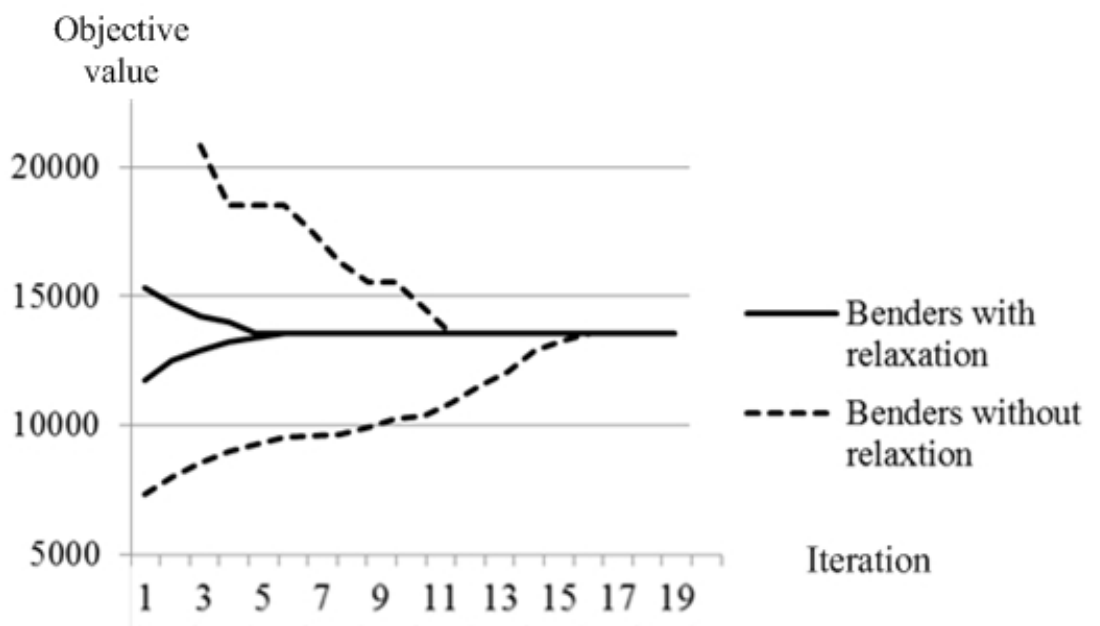

Figure 8: UB and LB values for Benders with and without relaxation constraints (21) to (25)

Table 2: Computational results

\begin{tabular}{|c|c|c|c|c|c|c|c|c|c|c|}
\hline \multirow{3}{*}{$\mathrm{I}, \mathrm{K}$} & \multicolumn{5}{|c|}{$S_{a v e}=25 \%$} & \multicolumn{5}{|c|}{$S_{\text {ave }}=50 \%$} \\
\hline & \multicolumn{3}{|c|}{ CPLEX } & \multicolumn{2}{|c|}{ Benders } & \multicolumn{3}{|c|}{ CPLEX } & \multicolumn{2}{|c|}{ Benders } \\
\hline & aveT & num & gap & aveT & gap & aveT & num & gap & aveT & gap \\
\hline 10,5 & 86.36 & 10 & 0.09 & 14.70 & 0.50 & 24.06 & 10 & 0.12 & 20.55 & 0.28 \\
\hline 10,7 & 58.05 & 10 & 0.04 & 17.11 & 0.21 & 32.90 & 10 & 0.00 & 21.03 & 0.31 \\
\hline 15,5 & 571.20 & 10 & 0.39 & 29.73 & 0.24 & 149.71 & 10 & 0.35 & 53.25 & 0.29 \\
\hline 15,7 & 385.97 & 10 & 0.64 & 37.18 & 0.35 & 175.78 & 10 & 0.33 & 65.93 & 0.57 \\
\hline 20,5 & 1204.32 & 6 & 2.30 & 55.56 & 0.54 & 1324.17 & 2 & 3.36 & 81.33 & 0.40 \\
\hline 20,7 & 1117.89 & 8 & 1.43 & 68.92 & 0.52 & - & 0 & 3.59 & 94.39 & 0.65 \\
\hline
\end{tabular}

putations can be terminated within the restrictions of the two above stopping criterions, and column gap reports the average relative gap in percentage. In both the methods, the number of materials has the greatest influence on the solution time. For instances with the same number of material, those who have more empty slots are generally easier to be solved by CPLEX. The results show that the proposed algorithm outperforms the standard solver in terms of solution time and the relative gap, and can solve all the instances to optimal.

\section{Conclusion}

In this paper, we considered the storage space allocation problem motivated by an inland bulk material stockyard. We addresses various practical concerns of the storage space 
allocation in bulk material stockyard, such as scattered small field and realistic stockyard structure. A novel mathematical formulation is developed based on the idea of partitioning the storage space into slots. We identify two pairs of strong relationships among different groups of decision making processes, and then accordingly apply a Benders Decomposition based approach to decompose the problem and solve it efficiently. The computational tests on instances show that our approach is able to solve the realistic problem in a considerably short time. The formulation and approach proposed in this paper is also valid for other stockyards that are operated similarly.

Future studies can consider the extensions of our model and solution method. Consider the stockyard in a bulk cargo terminal, the seagoing vessels are carrying materials in large volume. Thus the planning horizon will be longer, and we have to consider that the stockyard might not always have sufficient space prior to the arrival of new materials. In this case, we have to determine the yard clearance by the reclaiming operations in a longer planning horizon. The concept of updatable border slot proposed in this paper is still applicable but needs to be adjusted. Also, we might have to determine multiple storage locations for one material carried by one vessel due to the large volume.

\section{Acknowledgments}

This research was supported by the Fund for Innovative Research Groups of the National Natural Science Foundation of China (71621061), the Major International Joint Research Project of the National Natural Science Foundation of China (71520107004), the Major Program of National Natural Science Foundation of China (71790614), the 111 Project (B16009), and the Fundamental Research Funds for the Central Universities in China (N182803004).

\section{References}

Masatoshi Ago, Tatsushi Nishi, and Masami Konishi. Simultaneous optimization of storage allocation and routing problems for belt-conveyor transportation. Journal of Advanced Mechanical Design Systems $\&$ Manufacturing, 28(5):439-444, 2007.

Jasem Al-Hammadi and Ali Diabat. An integrated berth allocation and yard assignment problem for bulk ports: Formulation and case study. RAIRO - Operations Research, 50 (4):267-284, 2017.

Enrico Angelelli, Thomas Kalinowski, Reena Kapoor, and Martin W. P. Savelsbergh. A reclaimer scheduling problem arising in coal stockyard management. Journal of Scheduling, 19(5):563-582, 2016. 
Soudagar A K Irfan Babu, Saurabh Pratap, Geet Lahoti, Kiran J Fernandes, Manoj K Tiwari, Matthew Mount, and Yu Xiong. Minimizing delay of ships in bulk terminals by simultaneous ship scheduling, stockyard planning and train scheduling. Maritime Economics \& Logistics, 17(4):464-492, 2015.

Victor Hugo Barros, Tarcsio Souza Costa, Alexandre C. M. Oliveira, and Luiz A. N. Lorena. Model and heuristic for berth allocation in tidal bulk ports with stock level constraints. Computers \& Industrial Engineering, 60(4):606-613, 2011.

Mohammad Bazzazi, Nima Safaei, and Nikbakhsh Javadian. A genetic algorithm to solve the storage space allocation problem in a container terminal. Computers $\&$ Industrial Engineering, 56(1):44-52, 2009.

J. F. Benders. Partitioning procedures for solving mixed-variables programming problems. Numerische Mathematik, 4(1):238-252, Dec 1962.

Natashia Boland, Damon Gulczynski, and Martin Savelsbergh. A stockyard planning problem. EURO Journal on Transportation and Logistics, 1(3):197-236, Sep 2012.

Andre A. Cire, Elvin Coban, and John N. Hooker. Logic-based benders decomposition for planning and scheduling: a computational analysis. Knowledge Engineering Review, 31 (5):440-451, 2016.

Ahmed W A Hammad, Ali Akbarnezhad, and David Rey. Sustainable urban facility location: Minimising noise pollution and network congestion. Transportation Research Part E Logistics \& Transportation Review, 107:38-59, 2017.

J. N. Hooker and G. Ottosson. Logic-based benders decomposition. Mathematical Programming, 96(1):33-60, 2003.

Dayong $\mathrm{Hu}$ and Zhenqiang Yao. Stacker-reclaimer scheduling in a dry bulk terminal. International Journal of Computer Integrated Manufacturing, 25(11):1047-1058, 2012.

Thomas Kalinowski, Reena Kapoor, and Martin W. P. Savelsbergh. Scheduling reclaimers serving a stock pad at a coal terminal. Journal of Scheduling, 20(1):1-17, 2017.

Shuqin Li and Shuai Jia. A benders decomposition algorithm for the order fulfilment problem of an e-tailer with a self-owned logistics system. Transportation Research Part E: Logistics and Transportation Review, 122:463-480, 2019.

G. Lodewijks, D. L. Schott, and J. A. Ottjes. Modern dry bulk terminal design. Bulk Solids Handling, 27(6):364-376, 2007. 
Gustavo Campos Menezes, Geraldo Robson Mateus, and Martn Gmez Ravetti. A branch and price algorithm to solve the integrated production planning and scheduling in bulk ports. European Journal of Operational Research, 258(3):926 - 937, 2017.

Soo Suh Min, Yung Jae Lee, and Young Kwan Ko. A two-level hierarchical approach for raw material scheduling in steelworks. Engineering Applications of Artificial Intelligence, 10(5):503-515, 1997.

Tom Robenek, Nitish Umang, Michel Bierlaire, and Stefan Ropke. A branch-and-price algorithm to solve the integrated berth allocation and yard assignment problem in bulk ports. European Journal of Operational Research, 235(2):399-411, 2014.

Defeng Sun, Lixin Tang, and Roberto Baldacci. A benders decomposition-based framework for solving quay crane scheduling problems. European Journal of Operational Research, 273(2):504-515, 2019.

Lixin Tang, Wei Jiang, Jiyin Liu, and Yun Dong. Research into container reshuffling and stacking problems in container terminal yards. IIE Transactions, 47(7):751-766, 2015.

Lixin Tang, Defeng Sun, and Jiyin Liu. Integrated storage space allocation and ship scheduling problem in bulk cargo terminals. IIE Transactions, 48(5):428 -439, 2016.

Nitish Umang, Michel Bierlaire, and Ilaria Vacca. Exact and heuristic methods to solve the berth allocation problem in bulk ports. Transportation Research Part E:Logistics and Transportation Review, 54(7):14-31, 2013.

Ozgur Unsal and Ceyda Oguz. An exact algorithm for integrated planning of operations in dry bulk terminals. Transportation Research Part E: Logistics and Transportation Review, 126:103 - 121, 2019.

Teus Van Vianen, Jaap Ottjes, and Gabriel Lodewijks. Simulation-based determination of the required stockyard size for dry bulk terminals. Simulation Modelling Practice 86 Theory, 42(3):119-128, 2014.

Yat Wah Wan, Jiyin Liu, and Pei Chun Tsai. The assignment of storage locations to containers for a container stack. Naval Research Logistics, 56(8):699-713, 2010.

Jianbin Xin, Rudy R. Negenborn, and Teus Van Vianen. A hybrid dynamical approach for allocating materials in a dry bulk terminal. IEEE Transactions on Automation Science G Engineering, 99:1-11, 2018.

Mingzhu Yu and Xiangtong Qi. Storage space allocation models for inbound containers in an automatic container terminal. European Journal of Operational Research, 226(1): $32-45,2013$. 
Chuqian Zhang, Jiyin Liu, Yat Wah Wan, Katta G Murty, and Richard J Linn. Storage space allocation in container terminals. Transportation Research Part B Methodological, 37(10):883-903, 2003. 